\title{
On Vertices of Degree $n$ in Minimally n-Edge-Connected Graphs
}

\author{
W. MADER \\ Institut für Mathematik, \\ Universität Hannover, \\ Welfengarten 1, D-30167 Hannover
}

Received 1 October 1992; revised 22 September 1993

\begin{abstract}
Let $G$ be a minimally $n$-edge-connected finite simple graph with vertex number $|G| \geq$ $2 n+2+[3 / n\rfloor$ and let $n \geq 3$ be odd. It is proved that the number of vertices of degree $n$ in $G$ is at least $\left(\left(n-1-\epsilon_{n}\right) /(2 n+1)\right)|G|+2+2 \epsilon_{n}$, where $\epsilon_{n}=(3 n+3) /\left(2 n^{2}-3 n-3\right)$, and that for every $n \equiv 3(\bmod 4)$ this lower bound is attained by infinitely many minimally $n$-edge-connected finite simple graphs.
\end{abstract}

\section{Introduction}

Whereas the number $|G|_{n}$ of vertices of degree $n$ that a minimally $n$-connected graph $G$ must have, dependent on the number $|G|$ of vertices of $G$, is almost exactly known (see [8]), the corresponding problem for minimally $n$-edge-connected simple graphs is far from being settled. It was shown in [4] that every minimally $n$-edge-connected finite graph has two vertices of degree $n$ (see also Lemma 13 in [7]), which of course is best possible for every vertex number. But for simple graphs, i.e., graphs without multiple edges, this was improved in [5]: every minimally $n$-edge-connected finite simple graph has at least $n+1$ vertices of degree $n$. In [6] it was proved that, for every $n \neq 1,3$ there exists a $c_{n}>0$ such that every minimally $n$-edge-connected finite simple graph $G$ has at least $c_{n}|G|$ vertices of degree $n$. For $n=1,3$ such a result does not hold, as the example in Figure 1 shows for $n=3$. The value of the constant $c_{n}$ was improved in [1] and [2], and a rather good estimate for $|G|_{n}$ was given quite recently by Cai Mao-Cheng [3].
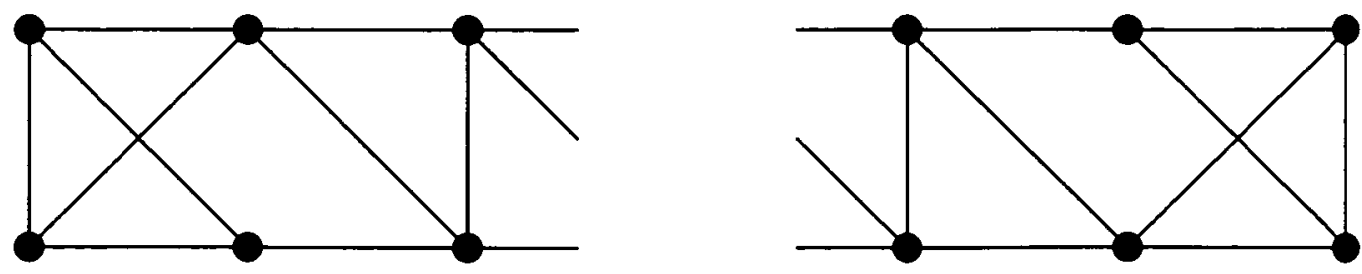

Figure 1 
Theorem. ([3]) If $G$ is a minimally n-edge-connected finite simple graph,

$$
\begin{aligned}
|G|_{n} & \geq \frac{n-\frac{1}{2}}{2 n+3}|G|+\frac{7 n+\frac{1}{2}}{2 n+3} \quad \text { for even } n \geq 6 \\
\text { and }|G|_{n} & \geq \frac{n-\frac{1}{2}}{2 n+5}|G|+\frac{10 n+1}{2 n+5} \quad \text { for odd } n \geq 7
\end{aligned}
$$

The lower bounds in this theorem are much better than the bounds given before, but they are not best possible. The main result of our paper will be the following best possible linear bound for $n \equiv 3 \quad(\bmod 4)$.

If $G$ is a minimally $n$-edge-connected finite simple graph containing at least $2 n+2+\lfloor 3 / n\rfloor$ vertices and if $n \geq 3$ is odd,

$$
|G|_{n} \geq \frac{n-1-\frac{3 n+3}{2 n^{2}-3 n-3}}{2 n+1}|G|+2+2 \cdot \frac{3 n+3}{2 n^{2}-3 n-3}
$$

holds and, for every $n \equiv 3 \quad(\bmod 4)$, the lower bound is attained by infinitely many simple graphs $G$.

First we will clarify our terminology and notation. A graph $G=(V(G), E(G))$ is always finite here. It may have multiple edges, but no loops. A simple graph is a graph without multiple edges. The set of edges joining the vertices $x$ and $y$ in $G$ is denoted by $[x, y]_{G}$. When we consider a special edge between $x$ and $y$, we write $[x, y]$ for this edge. For vertex sets or subgraphs $H_{1}, H_{2}$ of $G$, set

$$
\left[H_{1}, H_{2}\right]_{G}:=\bigcup_{\substack{x \in H_{1} \\ y \in H_{2}}}[x, y]_{G},
$$

using $x \in G$ instead of $x \in V(G)$. For $X \subseteq V(G)$, define $d_{G}(X):=\left|[X, V(G)-X]_{G}\right|$. For $x \in G$, we define $d_{G}(x):=d_{G}(\{x\})$ and $N_{G}(x):=\left\{y \in V(G):[x, y]_{G} \neq \emptyset\right\}$. The order of an edge $[x, y]$ in $G$ is $\min \left\{d_{G}(x), d_{G}(y)\right\}$ and its multiplicity is $\left|[x, y]_{G}\right|$. For $A \subseteq V(G)$, let $G(A):=G-(V(G)-A)$ be the subgraph induced by $A$ and let $C(x ; G)$ be the component of $G$ containing $x \in G$. Furthermore, we define $V_{n}(G):=\left\{x \in G: d_{G}(x)=n\right\}$, $V_{>n}(G):=\left\{x \in G: d_{G}(x)>n\right\},|G|_{n}:=\left|V_{n}(G)\right|$ and $|G|_{>n}:=\left|V_{>n}(G)\right|$. For disjoint sets $A$ and $B$, and a positive integer $k$, the graph $K_{A, B}^{k}$ is determined by $V\left(K_{A, B}^{k}\right):=A \cup B$ and $\left|[a, b]_{K_{A, B}^{k}}\right|:=k$ for every $a \in A$ and $b \in B$. In the notation of this paragraph, in general, we write $x$ instead of $\{x\}$, and we omit a subscript for the graph considered if it is obvious from the context which graph is meant.

The edge-connectivity number of a graph $G$ is denoted by $\lambda(G)$. A cut of $G$ is an $S \subseteq E(G)$ with $|S|=\lambda(G)$, such that $G-S$ is disconnected. If $\lambda(G)>0$ and $S$ is a cut of $G$, then $G-S$ has exactly two components, the components of the cut. A graph $G$ with $\lambda(G)=n$, but $\lambda(G-e)<n$ for every $e \in E(G)$ is called minimally $n$-edge-connected. It is well known that an $n$-edge-connected graph $G$ is minimally $n$-edge-connected if and only if every edge $e \in E(G)$ is contained in a cut of $G$.

In spite of being interested in $|G|_{n}$ for simple graphs $G$, we admit some multiple edges. Let $\mathscr{F}_{n}$ denote the class of all minimally $n$-edge-connected graphs with $|G| \geq 3$ that 

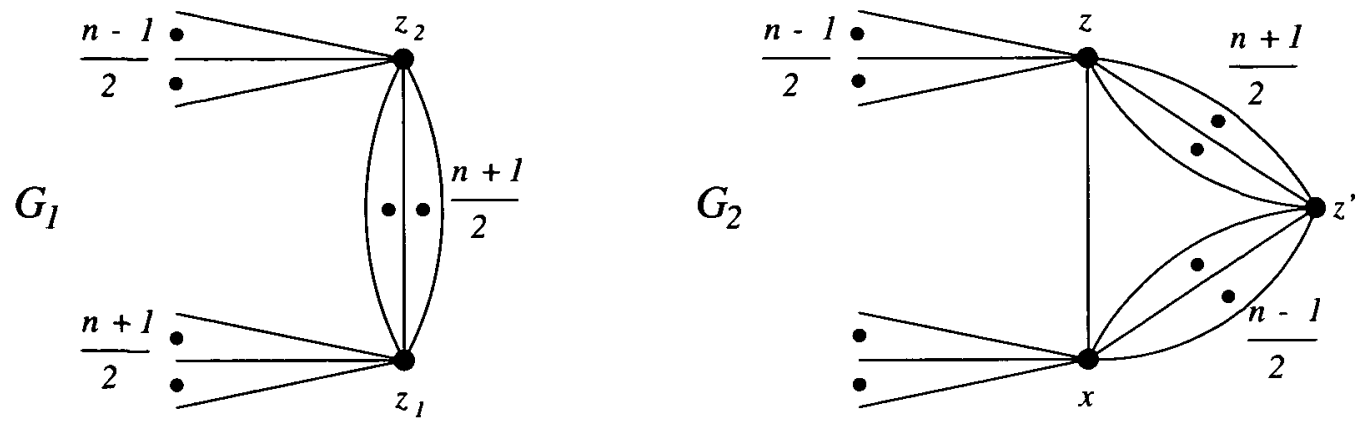

Figure 2

contain a vertex $z$ of degree $n$ such that $G-z$ is simple. By Lick's result [4], $|G|_{n} \geq 2$ for every $G \in \mathscr{F}_{n}$ and every minimally $n$-edge-connected graph is in $\mathscr{F}_{n}$.

Lemma 1.1. If $G \in \mathscr{F}_{n}$, then $|G| \geq n+2$ or $G$ is isomorphic to the complete graph $K_{n+1}$.

Proof. By the definition of $\mathscr{F}_{n}$, there is a $z \in V_{n}(G)$ such that $G-z$ is simple. Let us assume $|G| \leq n+1$. Then $[x, z]_{G} \neq \emptyset$ for every $x \in G-z$, since $G-z$ is simple. If there were an $x \in V_{>n}(G)$, this would imply the existence of a $y \in N(x)$ with $[y, z]_{G}=\emptyset$, since $d(x)>d(z)$ and $G-z$ is simple. This contradiction shows that $V_{>n}(G)=\emptyset$. Consider $x \in G-z$ and let $m:=\left|[x, z]_{G}\right|$; then $1 \leq m<n$, since $V_{n}(G)=V(G)$ and $|G| \geq 3$. For each $y \in N(x)-\{z\}$ we have $\left|[y, z]_{G}\right|=1$, because $\left|[y, z]_{G}\right| \geq 2$ would imply $d(z)>n$, since $G-z$ is simple. Since $N(x)-\{z\} \neq \emptyset$, we see that $m=1$; hence $|G|=n+1$ and $G$ is complete.

For the rest of the paper we assume $n \geq 3$ to be odd. We now show that in certain cases we can construct from $G \in \mathscr{F}_{n}$ a new member of $\mathscr{F}_{n}$ with one more vertex, but with the same number of vertices of degree $n$.

Procedure I. (PRI) Suppose $G \in \mathscr{F}_{n}$ and let $z \in V_{n}(G)$ be such that $G-z$ is simple. We assume that for all $x \in G,\left|[z, x]_{G}\right| \leq 2$ holds. Then we can find $Z_{1}, Z_{2} \subseteq N(z)$ with $Z_{1} \cup Z_{2}=N(z)$ and $\left|Z_{1}\right|=(n+1) / 2=\left|Z_{2}\right|+1$ such that $x \in Z_{1} \cap Z_{2}$ if and only if $\left|[z, x]_{G}\right|=2$. Then $G_{1}$ may be obtained from $G-z$ by adding vertices $z_{1} \neq z_{2}$, an edge between $z_{i}$ and each $x \in Z_{i}$, for $i=1,2$, and $(n+1) / 2$ edges between $z_{1}$ and $z_{2}$ (see Figure 2).

It is easily seen that $G_{1} \in \mathscr{F}_{n}$ also and that $\left|G_{1}\right|_{n}=|G|_{n}$.

Procedure II. (PRII) Suppose $G \in \mathscr{F}_{n}$ and let $z \in V_{n}(G)$ be such that $G-z$ is simple. We assume that there is an $x \in N(z)$ such that $\left|[z, x]_{G}\right|=(n+1) / 2$, but that $\left|\left[z, x^{\prime}\right]_{G}\right|=1$ for all $x^{\prime} \in N(z)-\{x\}$. Then $G_{2}$ may be obtained from $G$ by deleting $(n-1) / 2$ of the edges $[z, x]_{G}$ and adding a vertex $z^{\prime}$ together with $(n-1) / 2$ edges between $z^{\prime}$ and $x$ and $(n+1) / 2$ edges between $z^{\prime}$ and $z$ (see Figure 2).

Again, $G_{2} \in \mathscr{F}_{n}$ and $\left|G_{2}\right|_{n}=|G|_{n}$ hold. 


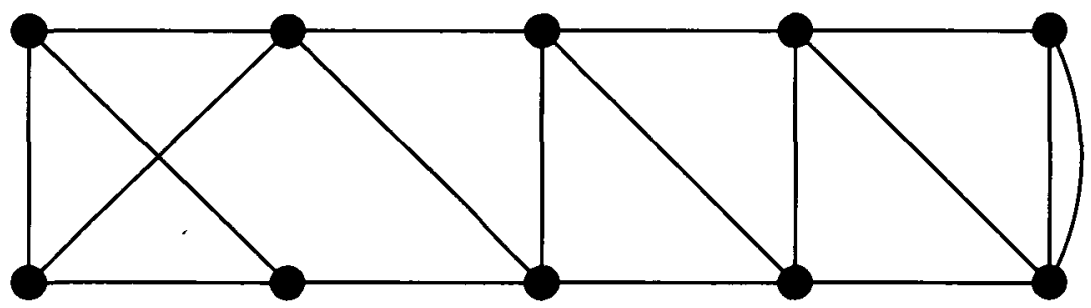

Figure 3

\begin{tabular}{ccccccc}
\multicolumn{8}{c}{ Table 1 } \\
\hline \hline$n$ & 3 & 5 & 7 & 9 & 11 & 13 \\
\hline$\epsilon_{n}$ & 2 & $\frac{9}{16}$ & $\frac{12}{37}$ & $\frac{5}{22}$ & $\frac{18}{103}$ & $\frac{21}{148}$ \\
\hline \hline
\end{tabular}

Starting from a simple graph $G \in \mathscr{F}_{n}$, we can first apply PRI to get $G_{1} \in \mathscr{F}_{n}$, and then apply PRII to $G_{1}$ to obtain $G_{2} \in \mathscr{F}_{n}$ with $\left|G_{2}\right|_{n}=|G|_{n}$. For $n \geq 5$ we cannot continue in this way, as neither PRI nor PRII can be applied to $G_{2}$. For $n=3$, however, we can apply PRI to $G_{2}$, and so on. (For $n=3$, if PRII is applicable to a graph, so is PRI, and they give the same result.) In this way, we can construct graphs in $\mathscr{F}_{3}$ of every order $(\geq 4)$ with exactly 4 vertices of degree 3 (in a similar way to Figure 1). For instance, starting from $K_{4}$ and applying PRI six times, we get the graph in Figure 3. This explains why the case $n=3$ is exceptional.

Since we are seeking an estimate of the form $|G|_{n} \geq f(|G|)$ for $G \in \mathscr{F}_{n}$, the above considerations suggest that we have to reduce the vertex number for those $G \in \mathscr{F}_{n}$ that are producible by PRI or PRII.

Definition 1. For $G \in \mathscr{F}_{n}$, we define $\delta(G) \in\{0,1,2\}$ as follows. If there is a $z \in G$ with $N(z)=\left\{x_{1}, x_{2}\right\}$ such that $\left|\left[z, x_{1}\right]_{G}\right|=(n-1) / 2$ and $\left|\left[z, x_{2}\right]_{G}\right|=(n+1) / 2$, then $\delta(G):=2$. If no such $z$ exists, but there is an edge of multiplicity exceeding $n / 2$, then $\delta(G):=1$. Otherwise $\delta(G):=0$.

For odd $n \geq 3$ we define

$$
\epsilon_{n}:=\frac{3 n+3}{2 n^{2}-3 n-3}
$$

It is easily seen that the sequence $\left(\epsilon_{n}\right)$ is strictly decreasing; the first few values are displayed in Table 1.

We now give constructions for $G \in \mathscr{F}_{n}$ having a small number of vertices of degree $n$. Let us start from the complete bipartite graph $H:=K_{n, n+1} \in \mathscr{F}_{n}$. Obviously $|H|_{n}=$ $\left(\left(n-1-\epsilon_{n}\right) /(2 n+1)\right)|H|+2+\epsilon_{n}$. Applying PRI and PRII successively to $H$, we get an $H^{\prime} \in \mathscr{F}_{n}$ with $\delta\left(H^{\prime}\right)=2$ and $\left|H^{\prime}\right|_{n}=\left(\left(n-1-\epsilon_{n}\right) /(2 n+1)\right)\left(\left|H^{\prime}\right|-\delta\left(H^{\prime}\right)\right)+2+\epsilon_{n}$. Consider a $G_{0} \in \mathscr{F}_{n}$ with $\delta\left(G_{0}\right)=2$ and $\left|G_{0}\right|_{n}=\left(\left(n-1-\epsilon_{n}\right) /(2 n+1)\right)\left(\left|G_{0}\right|-\delta\left(G_{0}\right)\right)+2+\epsilon_{n}$. By means of $G_{0}$, we now construct a graph $G \in \mathscr{F}_{n}$ with $|G|=((n-1) / 2)\left|G_{0}\right|+1$ and $|G|_{n}=\left(\left(n-1-\epsilon_{n}\right) /(2 n+1)\right)|G|+2+\epsilon_{n}$.

Let $G_{1}, \ldots, G_{k}$ be $k:=(n-1) / 2$ disjoint copies of $G_{0}$. Since $\delta\left(G_{i}\right)=2$, there is a vertex $z_{i} \in G_{i}$ with $N\left(z_{i}\right)=\left\{x_{i}, y_{i}\right\}$ such that $\left|\left[z_{i}, x_{i}\right]_{G_{i}}\right|=(n+1) / 2$ and $\left|\left[z_{i}, y_{i}\right]_{G_{i}}\right|=(n-1) / 2$ 


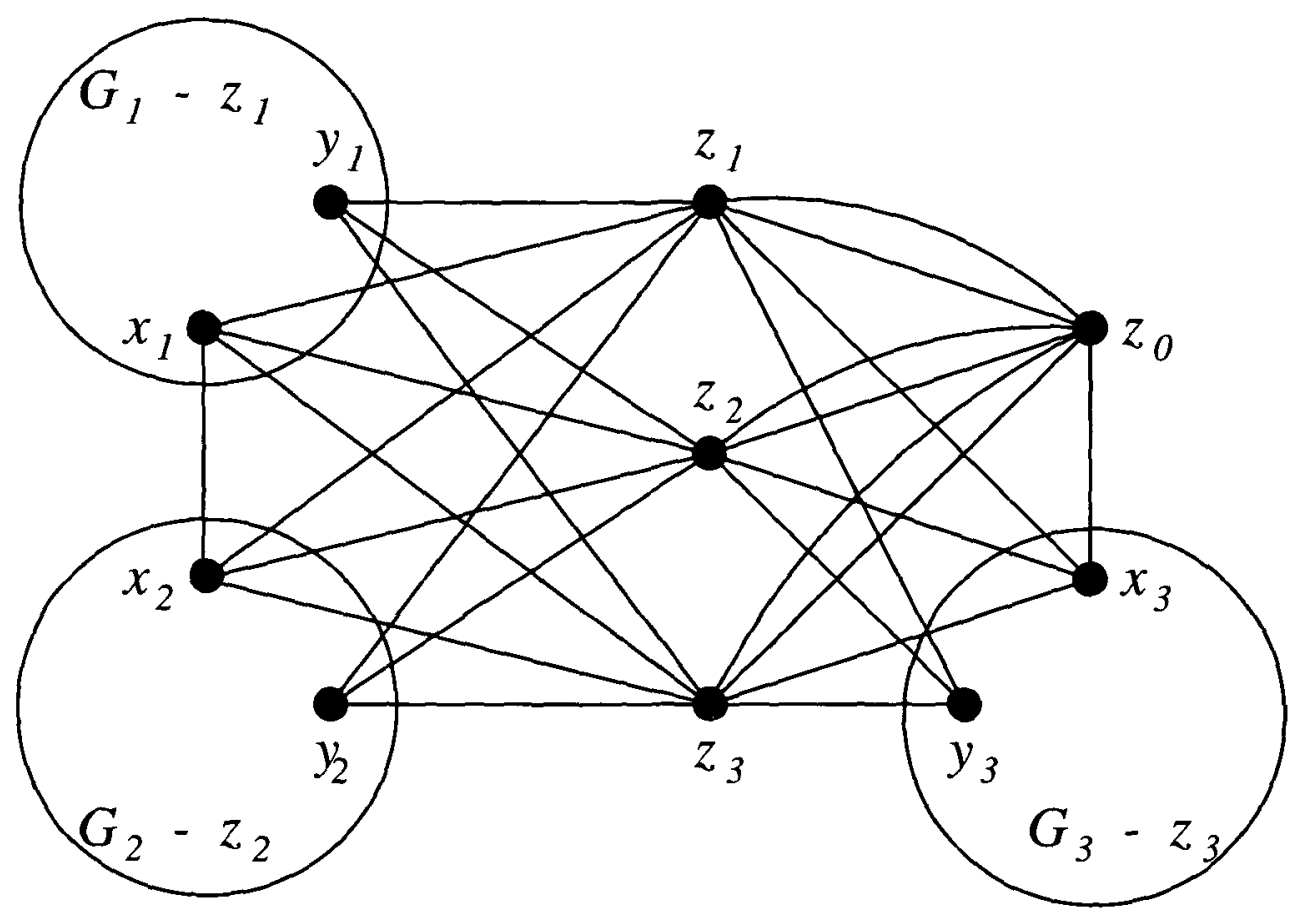

Figure 4

hold for each $i=1, \ldots, k$. Choose a further vertex $z_{0}$ and define a graph $G$ as follows (see Figure 4 for the case $n=7$ ):

$$
V(G):=\bigcup_{i=1}^{k} V\left(G_{i}\right) \cup\left\{z_{0}\right\}
$$

and

$$
\begin{gathered}
E(G):=\bigcup_{i=1}^{k} E\left(G_{i}-z_{i}\right) \cup E\left(K_{\left\{z_{1}, \ldots, z_{k}\right\}, \bigcup_{i=1}^{k}\left\{x_{i}, y_{i}\right\}}\right) \cup E\left(K_{z_{0},\left\{z_{1}, \ldots, z_{k}\right\}}^{2}\right) \\
\cup\left\{\left[x_{2 i-1}, x_{2 i}\right]: i=1, \ldots,\lfloor k / 2\}\right\} \cup\{e\},
\end{gathered}
$$

where, for $k$ odd, $e:=\left[z_{0}, x_{k}\right]$, and for $k$ even, $e$ is a further edge (not contained in $E\left(K_{z_{0},\left\{z_{1}, \ldots, z_{k}\right\}}^{2}\right)$ ) between $z_{0}$ and $z_{1}$. (Note that, for $n=3$, this construction provides the same graph as application of PRI.)

It is easily checked that $G \in \mathscr{F}_{n}$ and that

$$
\begin{aligned}
|G|_{n} & =\frac{n-1}{2}\left(\left|G_{0}\right|_{n}-1\right)+1 \\
& =\frac{n-1-\epsilon_{n}}{2 n+1}(|G|-n)+\frac{n-1}{2}\left(1+\epsilon_{n}\right)+1 \\
& =\frac{n-1-\epsilon_{n}}{2 n+1}|G|+2+\epsilon_{n},
\end{aligned}
$$




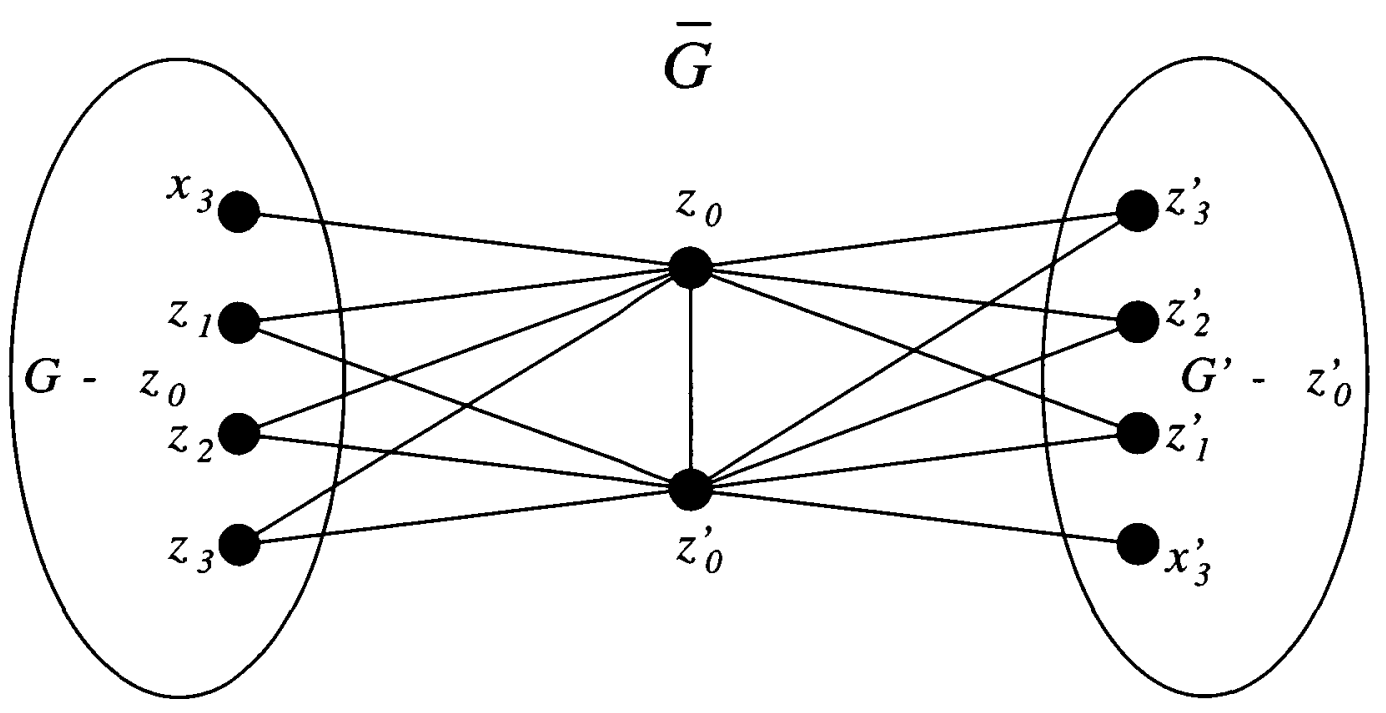

Figure 5

since $\epsilon_{n}$ was determined from the equation

$$
\frac{n-3}{2}\left(1+\epsilon_{n}\right)=n \frac{n-1-\epsilon_{n}}{2 n+1} \text {. }
$$

For $k$ even, we cannot apply PRI to $G$, since $\left|\left[z_{0}, z_{1}\right]_{G}\right|=3$, but for $k$ odd, we can apply PRI, PRII and the above construction successively, and so on. For $k$ odd, we get in this way graphs $G \in \mathscr{F}_{n}$ of arbitrarily large order with $\delta(G)=0$ for $n>3$ and with $|G|_{n}=\left(\left(n-1-\epsilon_{n}\right) /(2 n+1)\right)|G|+2+\epsilon_{n}$. Hence for $n \equiv 3 \quad(\bmod 4)$, the inequality $|G|_{n} \geq\left(\left(n-1-\epsilon_{n}\right) /(2 n+1)\right)(|G|-\delta(G))+2+\epsilon_{n}$ for $G \in \mathscr{F}_{n}$ would be best possible. The aim of this paper is to prove this inequality for all odd $n \geq 3$.

Theorem 1.1. For odd $n \geq 3$ and $G \in \mathscr{F}_{n}$,

$$
|G|_{n} \geq \frac{n-1-\epsilon_{n}}{2 n+1}(|G|-\delta(G))+2+\epsilon_{n}
$$

holds. For every $n \equiv 3(\bmod 4)$, there are $G \in \mathscr{F}_{n}$ of arbitrarily large order for which equality holds in (2).

The graphs constructed to give equality in (2) have edges of multiplicity two. But how sharp is the above estimate for simple $G \in \mathscr{F}_{n}$ ? For $n \equiv 3 \quad(\bmod 4)$, let us consider a $G \in \mathscr{F}_{n}$ with $|G|_{n}=\left(\left(n-1-\epsilon_{n}\right) /(2 n+1)\right)|G|+2+\epsilon_{n}$ provided by the above construction. Thus $z_{0}$ is a vertex of degree $n$ in $G$ with $\left|\left[z_{0}, z_{i}\right]_{G}\right|=2$ for $i=1, \ldots, k$ and $\left|\left[z_{0}, x_{k}\right]_{G}\right|=1$. Take a disjoint copy $G^{\prime}$ of $G$, where the vertices $z_{0}^{\prime}, z_{1}^{\prime}, \ldots, z_{k}^{\prime}, x_{k}^{\prime}$ correspond to $z_{0}, z_{1}, \ldots$, $z_{k}, x_{k}$, respectively. The simple graph $\bar{G}$ is now defined as follows (see Figure 5 for the case $k=3): V(\bar{G}):=V(G) \cup V\left(G^{\prime}\right)$ and

$$
E(\bar{G}):=E\left(G-z_{0}\right) \cup E\left(G^{\prime}-z_{0}^{\prime}\right) \cup E\left(K_{\left\{z_{0}, z_{0}^{\prime}\right\}, \bigcup_{i=1}^{k}\left\{z_{i}, z_{i}^{\prime}\right\}}^{1}\right) \cup\left\{\left[z_{0}, x_{k}\right],\left[z_{0}^{\prime}, x_{k}^{\prime}\right],\left[z_{0}, z_{0}^{\prime}\right]\right\} .
$$


It is easy to see that $\bar{G} \in \mathscr{F}_{n}$ and that $|\bar{G}|_{n}=2\left(|G|_{n}-1\right)=\left(\left(n-1-\epsilon_{n}\right) /(2 n+1)\right)|\bar{G}|+$ $2+2 \epsilon_{n}$. So we have shown that in the case $n \equiv 3(\bmod 4)$ of the following theorem, the lower bound is attained by infinitely many simple graphs.

Theorem 1.2. For $n \geq 3$ odd and simple $G \in \mathscr{F}_{n}$ with $|G| \geq 2 n+2+\lfloor 3 / n\rfloor$,

$$
|G|_{n} \geq \frac{n-1-\epsilon_{n}}{2 n+1}|G|+2+2 \epsilon_{n} .
$$

For every $n \equiv 3(\bmod 4)$, there are simple graphs $G \in \mathscr{F}_{n}$ of arbitrarily large order for which equality holds in (3).

For $n=3$, the above inequality $|G|_{3} \geq 6$ for simple $G \in \mathscr{F}_{3}$ with $|G| \geq 9$ was proved on p. 403 of [6].

For the proof of Theorems 1.1 and 1.2, a result of Cai Mao-Cheng from [3] is essential, but before stating it, we need some further definitions.

Let $A \subseteq V(G)$ be non-empty and take $a \notin G-A$. We define the contraction $G / A$ by the following equations: $V(G / A):=(V(G)-A) \cup\{a\}, E(G / A-\{a\}):=E(G-A)$, and for every $x \in G-A,[a, x]_{G / A}:=[A, x]_{G}$. We say that $G / A$ arises from $G$ by contracting $A$ to a. For a non-empty subgraph $H \subseteq G$, we set $G / H:=G / V(H)$.

If a component $C$ of a cut in a minimally $n$-edge-connected $G$ is contracted, then $G / C$ is also minimally $n$-edge-connected, as is well known (see, for example, Lemma 2 of [7]). We now state the previously mentioned result of Cai Mao-Cheng (see Theorem 3 and Corollary 2 of [3])

Lemma 1.2. ([3]) Let $G$ be a minimally n-edge-connected graph. Then there is a tree $T$ and a surjection $f: V(G) \rightarrow V(T)$ with the following properties:

(a) for every $[x, y] \in E(T), C(x, y):=\left[f^{-1}(V(C(x ; T-[x, y]))), f^{-1}(V(C(y ; T-[x, y])))\right]_{G}$ is a cut in $G$;

(b) for every $e \in E(G)$ of order exceeding $n$, there is an edge $[x, y]$ of $T$ with $e \in C(x, y)$;

(c) for every $[x, y] \in E(T), C(x, y)$ contains an edge of order exceeding $n$ which is not contained in any $C\left(x^{\prime}, y^{\prime}\right)$ with $\left[x^{\prime}, y^{\prime}\right] \in E(T-[x, y])$.

Property (b) implies immediately that for every $x \in T, V_{>n}(G) \cap f^{-1}(x)$ is independent in $G$. On the other hand, (c) implies that $V_{>n}(G) \cap f^{-1}(x) \neq \emptyset$ for every $x \in T$ if $|T|>1$. For then we can choose an edge $[x, y] \in E(T)$, and by (c) there is an edge $[u, v] \in C(x, y)$ of order exceeding $n$ not contained in any other cut $C\left(x^{\prime}, y^{\prime}\right)$. Let us consider the path $P$ joining $f(u)$ and $f(v)$ in $T$ and an edge $\left[x^{\prime}, y^{\prime}\right] \in E(P)$. Then it follows that $[u, v] \in C\left(x^{\prime}, y^{\prime}\right)$, and hence by the choice of $[u, v], E(P)=\{[x, y]\}=\{[f(u), f(v)]\}$ and $f^{-1}(x) \cap\{u, v\} \neq \emptyset$. So we get the following corollary.

\section{Corollary 1.2. ([3])}

(1) For every $x \in T, V_{>n}(G) \cap f^{-1}(x)$ is independent in $G$.

(2) If $|T|>1$, then $V_{>n}(G) \cap f^{-1}(x) \neq \emptyset$ for every $x \in T$. 


\section{Proofs of Theorems 1.1 and 1.2}

We now proceed to the proof of our theorems. First we will show that Theorems 1.1 and 1.2 are true if $G \in \mathscr{F}_{n}$ has no edge of order exceeding $n$. For this we need the following (probably known) lemma.

Lemma 2.1. Let $G$ be a graph of minimum degree $m \geq 1$ with no edge of order exceeding m. Then

$$
|G|_{m} \geq \frac{m+1}{2 m+1}|G|>\frac{1}{2}|G|
$$

and

hold.

$$
|G|_{m} \geq \frac{1}{2}|G|+\frac{1}{2 m}|G|_{>m}
$$

Proof. Obviously, $(m+1)\left(|G|-|G|_{m}\right)=(m+1)|G|_{>m} \leq d_{G}\left(V_{>m}(G)\right) \leq m|G|_{m}$ holds, which implies the first inequality and $2 m|G|_{m} \geq m|G|+|G|_{>m}$, implying the second.

Lemma 2.2. Assume $G \in \mathscr{F}_{n}$ has no edge of order exceeding $n$. Then

$$
|G|_{n} \geq \frac{n-1-\epsilon_{n}}{2 n+1}|G|+2+\epsilon_{n}
$$

holds and if $|G| \geq 2 n+2+\lfloor 3 / n\rfloor$, we even have

$$
|G|_{n} \geq \frac{n-1-\epsilon_{n}}{2 n+1}|G|+2+2 \epsilon_{n}
$$

Proof. Let us first prove (4). Since $((n+1) /(2 n+1))|G|$ is not less than the righthand side of (4) for $|G| \geq 2 n+1$, by Lemma 2.1 , we can assume $|G| \leq 2 n$. Then $\left(\left(n-1-\epsilon_{n}\right) /(2 n+1)\right)|G|+2+\epsilon_{n} \leq n+1$ holds, so we may assume $|G|_{n} \leq n$. By the definition of $\mathscr{F}_{n}$, there is a $z \in V_{n}(G)$ such that $G-z$ is simple. Since $|G|_{n} \leq n$, we get $|G| \geq n+2$, and hence $|G|_{>n} \geq 2$ from Lemma 1.1. Choose any $x \in V_{>n}(G)$. Then $N(x) \subseteq V_{n}(G)$, since there is no edge of order exceeding $n$, and hence $\left|[x, z]_{G}\right| \geq 2$, since $|G|_{n} \leq n$. But $\left|[x, z]_{G}\right| \geq 2$ for all $x \in V_{>n}(G)$ implies $|G|_{>n} \leq(n-1) / 2$, hence $n \geq 5$ and $|G|=|G|_{n}+|G|_{>n} \leq n+(n-1) / 2 \leq 2 n-3$. If $|G|_{>n}=(n-1) / 2$, then there is an $x \in V_{>n}(G)$ with $\left|[x, z]_{G}\right| \leq 2$, which implies $|G|_{n} \geq|N(x)| \geq n$. But then $|G|_{n}=n \geq\left(\left(n-1-\epsilon_{n}\right) /(2 n+1)\right)|G|+2+\epsilon_{n}$, because we have $|G| \leq 2 n-3$ and because $4\left(n-1-\epsilon_{n}\right) /(2 n+1) \geq 1$ for $n \geq 5$. So we can assume $|G|_{>n} \leq(n-3) / 2$, which implies $n \geq 7$ and $|G|_{n} \geq n+2-(n-3) / 2=(n-3) / 2+5 \geq|G|_{>n}+5$. Hence we get $|G|_{n} \geq$ $|G| / 2+5 / 2 \geq\left(\left(n-1-\epsilon_{n}\right) /(2 n+1)\right)|G|+2+\epsilon_{n}$, because we have $\epsilon_{n} \leq 1 / 2$ for $n \geq 7$.

Let us now turn to (5). Consider $G \in \mathscr{F}_{n}$ with $|G| \geq 2 n+2$; then we may write $|G|=$ $2 n+1+k$ with $k \geq 1$. By Lemma $2.1,|G|_{n} \geq((n+1) /(2 n+1))|G|>n+1+k / 2$ and hence $|G|_{n} \geq n+2+\lfloor k / 2\rfloor$. So it suffices to show that $1+\lfloor k / 2\rfloor \geq\left(\left(n-1-\epsilon_{n}\right) /(2 n+1)\right) k+\epsilon_{n}$. This is obviously true for $n \geq 7$, since then $\epsilon_{n} \leq 1 / 2$, for $n=5$ and $k$ even, since $\epsilon_{5} \leq 1$, and for $n=3$ and $k \geq 2$. So it only remains (for the reader) to check that it is true for $n=5$ and $k=1$. 
Remark. One could conjecture that $|G|_{n} \geq n+1$ for $G \in \mathscr{F}_{n}$, but this is not true, as shown by the following example. Consider a positive integer $k$ with $k^{2} \leq n$ and choose $K_{k} \subseteq K_{n+1}$. Define $G$ by adding a vertex $z$ to $K_{n+1}-E\left(K_{k}\right)$ and at least $k$ edges from $z$ to each vertex of $K_{k}$ to give $z$ degree $n$. Then $G \in \mathscr{F}_{n}$, but $|G|_{n}=n+2-k$.

We now prove Theorem 1.1 by induction on $|G|$.

Proof of Theorem 1.1. Consider $G \in \mathscr{F}_{n}$ and $z \in G$ such that $G-z$ is simple. We suppose that (2) has been proved for all $G^{\prime} \in \mathscr{F}_{n}$ with $\left|G^{\prime}\right|<|G|$. By Lemma 2.2, we can assume that there is an edge in $G$ of order exceeding $n$. We choose a tree $T$ and a surjection $f: V(G) \rightarrow V(T)$ as described in Lemma 1.2. Since there is an edge in $G$ of order exceeding $n$, we have $|T| \geq 2$. Let $t_{0}:=f(z)$ and let $C_{0}:=f^{-1}\left(t_{0}\right)$. Since $z \in C_{0}$, and since $C_{0} \cap V_{>n}(G) \neq \emptyset$, by Corollary $1.2(2)$, we have $\left|C_{0}\right| \geq 2$, and, by Corollary 1.2 (1), $C_{0} \cap V_{>n}(G)$ is independent in $G$. Set $N_{T}\left(t_{0}\right)=\left\{t_{1}, \ldots, t_{d}\right\}$, where $d=d_{T}\left(t_{0}\right) \geq 1$, and $C_{i}:=f^{-1}\left(V\left(C\left(t_{i} ; T-\left[t_{0}, t_{i}\right]\right)\right)\right)$ for $i=1, \ldots, d$. Since $\left[C_{i}, G-C_{i}\right]_{G}=C\left(t_{0}, t_{i}\right)$ is a cut for $i=1, \ldots, d$, and since $C_{i} \cap V_{>n}(G) \neq \emptyset$, by Corollary $1.2(2)$, we have $\left|C_{i}\right| \geq 2$ for each $i=1, \ldots, d$.

For $i=1, \ldots, d$, the graph $G_{i}$ may arise from $G$ by contracting $G-C_{i}$ to $z_{i}$. Since $\left|C_{0}\right| \geq 2$, we have $\left|G_{i}\right|<|G|$ for $i=1, \ldots, d$. Since $\left|C_{i}\right| \geq 2$ and $\left[C_{i}, G-C_{i}\right]_{G}$ is a cut, we have $G_{i} \in \mathscr{F}_{n}$ for $i=1, \ldots, d$, because $z \in C_{0}$, so $G_{i}-z_{i}$ is simple. The graph $G_{0}$ may arise from $G$ by contracting $C_{i}$ to $c_{i} \in C_{i}$ for $i=1, \ldots, d$. Then $G_{0}$ is also minimally $n$-edgeconnected, because each $G\left(C_{i}\right)(i=1, \ldots, d)$ is a component of a cut of $G$, but in general $G_{0} \notin \mathscr{F}_{n}$. Let $\delta_{i}:=\delta\left(G_{i}\right)$ for $i=1, \ldots, d$, and let $\delta:=\delta(G)$. Without loss of generality, we may assume that the sequence $\left(\delta_{i}\right)$ is decreasing. Let $m:=\left|V_{n}(G) \cap C_{0}\right|=\left|G_{0}\right|_{n}-d$, and let $k:=\left|V_{>n}(G) \cap C_{0}\right|=\left|C_{0}\right|-m$. We have seen that $m \geq 1$ and $k \geq 1$.

By the induction hypothesis, we have

$$
\left|G_{i}\right|_{n} \geq \frac{n-1-\epsilon_{n}}{2 n+1}\left(\left|G_{i}\right|-\delta_{i}\right)+2+\epsilon_{n}
$$

for $i=1, \ldots, d$. Adding these inequalities, we get

$$
|G|_{n}=\sum_{i=1}^{d}\left(\left|G_{i}\right|_{n}-1\right)+m \geq \frac{n-1-\epsilon_{n}}{2 n+1}\left(|G|-\left|C_{0}\right|+d-\sum_{i=1}^{d} \delta_{i}\right)+d+d \epsilon_{n}+m .
$$

We will have finished when we have shown that the last expression is at least

$$
\left(\left(n-1-\epsilon_{n}\right) /(2 n+1)\right)(|G|-\delta)+2+\epsilon_{n} .
$$

But this is equivalent to the inequality

$$
\begin{aligned}
\left|G_{0}\right|_{n}=m+d & \geq \frac{n-1-\epsilon_{n}}{2 n+1}\left(\left|C_{0}\right|+d-s\right)+2-(d-1) \epsilon_{n} \\
& =\frac{n-1-\epsilon_{n}}{2 n+1}\left(\left|G_{0}\right|-s\right)+2-(d-1) \epsilon_{n},
\end{aligned}
$$

where $s$ denotes $2 d+\delta-\sum_{i=1}^{d} \delta_{i}$. By definition $1,0 \leq s \leq 2 d+\delta$. 
On the other side, we can apply Lemma 2.1 to $G_{0}$ and get $\left|G_{0}\right|_{n} \geq(1 / 2)\left|G_{0}\right|+1 / 2$. If even $\left|G_{0}\right|_{n} \geq(1 / 2)\left|G_{0}\right|+2$, then (6) is met. So we assume

$$
\frac{1}{2}\left(\left|C_{0}\right|+d\right)+\frac{1}{2} \leq m+d \leq \frac{1}{2}\left(\left|C_{0}\right|+d\right)+\frac{3}{2},
$$

say,

$$
m+d=\frac{1}{2}\left(\left|C_{0}\right|+d\right)+r \text { with } r \in\left\{\frac{1}{2}, 1, \frac{3}{2}\right\} .
$$

Inserting $\left|C_{0}\right|=m+k$, we get

$$
\left|G_{0}\right|_{n}=m+d=k+2 r .
$$

If $s \geq 4-2 r$, then by (7), $m+d=(1 / 2)\left(\left|C_{0}\right|+d+2 r-4\right)+2 \geq(1 / 2)\left(\left|C_{0}\right|+d-s\right)+2$, which implies (6). Hence we may assume

$$
s \leq 3-2 r .
$$

In particular, $s \leq 2$, and since $\left(\delta_{i}\right)$ is decreasing we get

$$
\begin{gathered}
\delta_{1}=\delta_{2}=\cdots=\delta_{d-2}=2, \text { and } \\
\text { for } d \geq 2, \delta_{d-1}+\delta_{d} \geq 1+2 r+\delta, \\
\quad \text { for } d=1, \delta_{1} \geq 2 r-1+\delta .
\end{gathered}
$$

Using (7), (6) turns out to be equivalent to the inequality

$$
\frac{3+2 \epsilon_{n}}{2(2 n+1)}\left(\left|C_{0}\right|+d\right)+\frac{n-1-\epsilon_{n}}{2 n+1} s+(d-1) \epsilon_{n} \geq 2-r .
$$

Let us suppose that $s=3-2 r$. Then, by eliminating $r,(11)$ can be transformed into

$$
\frac{3+2 \epsilon_{n}}{2(2 n+1)}\left(\left|C_{0}\right|+d-s\right)+(d-1) \epsilon_{n} \geq \frac{1}{2} \text {. }
$$

From the equation (1) for $\epsilon_{n}$, we can easily deduce

$$
\frac{3+2 \epsilon_{n}}{2(2 n+1)} \frac{2 n}{3}+\left(\frac{n}{3}-1\right) \epsilon_{n}=1
$$

This equation (12) shows that the last inequality will hold if we have $\left|C_{0}\right|+d-s \geq n / 3$ and $d \geq n / 6+1 / 2$. So we can assume:

$$
\text { If } s=3-2 r, \text { then }\left|C_{0}\right|+d-s<\frac{n}{3} \text { or } d<\frac{n}{6}+\frac{1}{2} .
$$

Now suppose that $d-1 \geq((n / 3)-1)(2-r)$. Using (7), we have that $\left|C_{0}\right|+d=$ $2 m+2 d-2 r \geq 2 m-2+(2 n / 3)(2-r) \geq(2 n / 3)(2-r)$ and then (12) shows that (11) holds. So we may assume

$$
d<\frac{n}{3}(2-r)+r-1 \text {. }
$$

This means that $d<(n+3) / 6$ for $r=3 / 2, d<n / 3$ for $r=1$ and $d \leq(n-3) / 2$ for $r=1 / 2$. 
For $\epsilon_{n}$, we have the lower bound

$$
\epsilon_{n}>\frac{3 n+3}{2 n^{2}-2 n-4}=\frac{3}{2(n-2)}>\frac{3}{2 n-1} .
$$

This and (12) imply

$$
\frac{3+2 \epsilon_{n}}{2(2 n+1)}\left(\frac{4 n-2}{3}-2 x\right)+x \epsilon_{n} \geq 1
$$

for all $x \geq 0$. So (11) is true if $\left|C_{0}\right|+d \geq((4 n-2) / 3)(2-r)-2 x$ and $d \geq x+1$ hold for any $x \geq 0$. This means that $(11)$ is met if $\left|C_{0}\right|+d \geq((4 n-2) / 3)(2-r)-2(d-1)$ holds. So we assume

$$
\left|C_{0}\right|+d<\frac{4 n-2}{3}(2-r)-2(d-1) .
$$

Then we have $\left|G_{0}\right|<(2 n-1) / 3$ for $r=3 / 2,\left|G_{0}\right|<(4 n-2) / 3$ for $r=1$ and $\left|G_{0}\right| \leq$ $2(n-d)$ for $r=1 / 2$.

We now distinguish the cases $r=3 / 2,1,1 / 2$.

Case $r=3 / 2$. Then by (8), $m+d=k+3$; hence $\left|C_{0}\right|+d=2 k+3$ and, using (16), this implies

$$
3 k+6 \leq n .
$$

From (9) we get $s=0$, hence $\delta_{d-1}=\delta_{d}=2$ and $\delta=0$. But $\delta_{i}=2$ implies that $\left|N_{G_{i}}\left(z_{i}\right)\right|=2$ and hence $\left|\left[x, C_{i}\right]_{G}\right| \leq 2$ for every $x \in G-\left(C_{i} \cup\{z\}\right)$. Therefore for $x \in V_{>n}\left(G_{0}\right) \neq \emptyset$ we get

$$
n+1 \leq d_{G}(x) \leq 2 d+m-1+\left|[x, z]_{G}\right|,
$$

since $V_{>n}\left(G_{0}\right)$ is independent. But $\left|[x, z]_{G}\right| \leq(n-1) / 2$, since $\delta=0$, which together with (14) gives

$$
2 d+m-1+\left|[x, z]_{G}\right|<k+2+\frac{n+3}{6}+\frac{n-1}{2} .
$$

This and (18) imply $k+2>(n+3) / 3$, which contradicts (17).

Case $r=1$. Then $m+d=k+2$ by (8), hence $\left|C_{0}\right|+d=2 k+2$, and (16) implies

$$
k<\frac{2 n-1}{3}-d \text {. }
$$

From (9) and (10) we get $s \leq 1, \delta_{d-1}=2$ and $\delta_{d} \geq 1+\delta$. From (14) we see that $n \geq 5$.

We will now deduce bounds for $\kappa_{0}:=d_{G_{0}}\left(V_{>n}\left(G_{0}\right)\right)$. Since $V_{>n}\left(G_{0}\right)$ is independent, we have

$$
\kappa_{0} \geq k(n+1) \text {. }
$$

As in the case $r=3 / 2$, we have $\left|\left[x, c_{i}\right]_{G_{0}}\right| \leq 2$ for $i=1, \ldots, d-1$ and $x \in C_{0}-\{z\}$. Since $\delta_{d} \geq 1$ there is a $y \in C_{d}$ with $\left|\left[y, G-C_{d}\right]_{G}\right| \geq(n+1) / 2$, and hence $\left|\left[C_{d}-\{y\}, C_{0}\right]_{G}\right| \leq$ $(n-1) / 2$. Since any edges in $G$ of multiplicity greater than one must be incident to $z$ and since $d(z)=n$, we get

$$
\kappa_{0} \leq k \cdot 2(d-1)+k+k(m-1)+\frac{n-1}{2}+n .
$$


If $\delta_{d}=2$ also, we even get

$$
\kappa_{0} \leq k \cdot 2 d+k(m-1)+n .
$$

The inequality (20), together with either (21) or (22), respectively, implies, by using $m=k+2-d$,

and

$$
k(n+1-d-k) \leq \frac{3 n-1}{2}
$$

$$
k(n-d-k) \leq n \text {, if } \delta_{d}=2 .
$$

If there is an edge $[z, x] \in E\left(G_{0}\right)$ with $x \notin V_{>n}\left(G_{0}\right)$, we have strict inequality in (21) and (22), and hence also in (23) and (24).

We will first show that we cannot have $k \geq 3$. For if the inequality $3(n+1-d-3) \leq$ $(3 n-1) / 2$ holds, we have $3 d \geq(3 n-11) / 2$ so $d \geq(3 n-9) / 6$ and hence by (14) we have $(n-3) / 2 \leq d \leq(n-1) / 3$. This implies that $n \leq 7$ and that if $n=7$ then $d=2$. This means that for $n \geq 9$ (and $d<n / 3$ ), (23) is neither true for $k=3$ nor for $k=n-2-d \geq 4$, and hence is not true for any $k \in\{3, \ldots, n-2-d\}$ by the concavity of the function $f(x)=x(n+1-d-x)$. Considering (19), we have shown that for $n \geq 9$, only $k=1$ and $k=2$ are possible. On the other hand, (19) implies that $n \geq 7$ if $k \geq 3$ and even that $n \geq 9$ if $k \geq 4$. So it only remains to consider the case $k=3$ and $n=7$, from which we had deduced above that $d=2$, contradicting (19). So only $k=1$ and $k=2$ are possible.

First let us assume that $k=2$. Then we have $\left|G_{0}\right|=\left|C_{0}\right|+d=2 k+2=6$ and $m+d=4$. From (23) we get

$$
d \geq \frac{n-3}{4},
$$

which implies $n \leq 15$, since $d \leq 3$. On the other hand, (19) implies $n \geq 7$. It is easily checked that (6) is true for $n=7$ (and $\left|G_{0}\right|=6, k=2$ ) if $s \geq 1$ or $d \geq 2$. But if $n=7$, we cannot have $s=0$ and $d=1$. For if $s=0$, it follows that $\delta_{d}=2$ and, by applying (24), we get $d \geq n / 2-2$ and hence $d \geq 2$ for $n=7$. So we may assume $n \geq 9$, and then $d \geq 2$ by (25). If $\delta_{d}=2$, we get

$$
\frac{n}{2}-2 \leq d<\frac{n}{3}
$$

from (24) and (14); hence $n=9$, since $d \leq 3$. But $n=9$ is inconsistent with (26). So we must have $\delta_{d}=1$, and hence $s=1$ and $\delta=0$. Then (13) is applicable and gives us

$$
d<\frac{n}{6}+\frac{1}{2}
$$

since the first inequality in (13) cannot hold, as $n \leq 15$. But (27) implies $d \leq 2$, hence $d=2$, and from (25) and (27) we get $n=11$. Since $\delta=0$, we have $\left|[x, z]_{G}\right| \leq(n-1) / 2$ for every $x \in G$, and hence $N_{G_{0}}(z) \nsubseteq V_{>n}\left(G_{0}\right)$. Then the inequality (23) must be strict, so $2(n-1-d) \leq(3 n-3) / 2$. But $d=2$, so this supplies the contradiction $n \leq 9$.

The remaining case is $k=1$, say $V_{>n}\left(G_{0}\right)=\{x\}$. Then $\left|C_{0}\right|+d=2 k+2=4$ and $\left|G_{0}\right|_{n}=m+d=3$ hold. Since $n$ is odd, $\left|G_{0}\right|_{n}=3$ implies $d(x) \geq n+2$. Suppose there is a $y \in V_{n}(G) \cap\left(C_{0}-\{z\}\right)$. Then $d=1$, and since $\delta_{1} \geq 1$, we have the estimate $(n+1)+(n-1) \leq d_{G}(\{x, y\}) \leq 2+(n-1) / 2+n$, which implies that $n \leq 3$. So we may assume that $m=1$ (hence $d=2$ ). Since $\delta_{1}=2$ and $\delta_{2} \geq 1$, we can find $y_{1}$, 
$y_{1}^{\prime} \in C_{1}$ and $y_{2} \in C_{2}$ such that $\left|\left[y_{1}, G-C_{1}\right]_{G}\right|=(n+1) / 2,\left|\left[y_{1}^{\prime}, G-C_{1}\right]_{G}\right|=(n-1) / 2$ and $\left|\left[y_{2}, G-C_{2}\right]_{G}\right| \geq(n+1) / 2$. So we have the estimate

$$
\begin{gathered}
(n-1)+\left(\frac{n+1}{2}-2\right)+\left(\frac{n+1}{2}-3\right) \leq \\
d_{G-\left\{y_{1}, y_{1}^{\prime}, y_{2}\right\}}(x)+\left|\left[y_{1},\left(C_{0} \cup C_{2}\right)-\left\{x, y_{2}\right\}\right]_{G}\right|+\left|\left[y_{2},\left(C_{0} \cup C_{1}\right)-\left\{x, y_{1}, y_{1}^{\prime}\right\}\right]_{G}\right| \\
\leq \frac{n-1}{2}+n,
\end{gathered}
$$

which implies $n \leq 9$. Then (13) shows that $s=1$ is impossible. So we have $s=0$, and hence $\delta_{2}=2$ and $\delta=0$. But then $n+2 \leq d(x) \leq 4+\left|[x, z]_{G}\right| \leq 4+(n-1) / 2$ implies $n \leq 3$. This contradiction completes the proof for the case $r=1$.

Case $r=1 / 2$. Then we have $m+d=k+1$ by (8), hence $\left|C_{0}\right|+d=2 k+1$, and from (16) we get

$$
k \leq n-d-1 \text {. }
$$

Furthermore, $s \leq 2$ by (9), and if $d \geq 2, \delta_{d-1}+\delta_{d} \geq 2+\delta$ by (10). Inequality (14) implies $n \geq 5$.

In a similar way to the case $r=1$, we deduce bounds for $\kappa_{0}:=d_{G_{0}}\left(V_{>n}\left(G_{0}\right)\right)$. We again have

$$
\kappa_{0} \geq k(n+1)
$$

We first give upper bounds for $\kappa_{1}:=\left|\left[V_{>n}\left(G_{0}\right), G-C_{0}\right]_{G}\right|$, which may be obtained by similar arguments to those used in the case $r=1$ :

$$
\text { If } \delta_{d-1}=2 \text { or } d=1 \text {, then } \kappa_{1} \leq k \cdot 2(d-1)+n \text {; }
$$

If $\delta_{d} \geq 1$ and $d \geq 2$, then $\kappa_{1} \leq k \cdot 2(d-1)+2 \frac{n-1}{2}$;

$$
\text { If }\left(\delta_{d-1}=2 \text { or } d=1\right) \text { and } \delta_{d} \geq 1 \text {, then } \kappa_{1} \leq k(2 d-1)+\frac{n-1}{2} \text {. }
$$

Since either $d=1$ or $\delta_{d-1}+\delta_{d} \geq 2$,

$$
\kappa_{0} \leq k \cdot 2(d-1)+n+k(m-1)+n
$$

follows from (30) and (31).

We can apply (32) to get:

$$
\text { If } s \leq 1 \text {, then } \kappa_{0} \leq k(2 d-1)+\frac{n-1}{2}+k(m-1)+n \text {. }
$$

Considering $m=k+1-d$, the inequality (29), together with (33) or (34) respectively, implies

$$
\begin{gathered}
k(n+3-d-k) \leq 2 n \\
\text { and } k(n+2-d-k) \leq \frac{3 n-1}{2} \text {, if } s \leq 1 \text { holds. }
\end{gathered}
$$

If there is a vertex $x \in V_{>n}\left(G_{0}\right)$ with $d(x) \geq n+2$, we have strict inequality in (29), and hence also in (35) and (36). 
For $k=4$ and $k=n-d-1$ we get, using (14), $k(n+3-d-k)=4(n-d-1) \geq$ $4(n-1-(n-3) / 2)>2 n$. So we see that (35) (with $d \leq(n-3) / 2)$ cannot be true for any $k$ between 4 and $n-d-1$, and hence we get $k \leq 3$ by (28).

First let us assume that $k=3$. Then we have $m+d=4$ and hence $d \leq 3$. From (35) and (14) we get $n / 3 \leq d \leq(n-3) / 2$. But these inequalities imply that $n=9$ and $d=3$, but then (13) shows that $s \leq 1$ and application of (36) with $k=d=3$ supplies the contradiction $n<8$. So $k=3$ is impossible.

Now suppose that $k=2$, and say $V_{>n}\left(G_{0}\right)=\left\{x_{1}, x_{2}\right\}$. Then we have $m+d=3$ and $\left|C_{0}\right|+d=5 .\left|G_{0}\right|_{n}=3$ implies that $d\left(x_{i}\right) \geq n+2$ for $i=1$ or $i=2$. Then the inequality in (35) is strict and implies $d \geq 2$, and hence $d=2$. Since $\delta_{1} \geq 1$, there is a $y \in C_{1}$ with $\left|\left[y, G-C_{1}\right]_{G}\right| \geq(n+1) / 2$. By $(10), \delta_{1}=2$ or $\delta_{2} \geq 1$. Now if $\delta_{1}=2$, there is a $y^{\prime} \in C_{1}$ with $\left|\left[y^{\prime}, G-C_{1}\right]_{G}\right|=(n-1) / 2$, and we get, as usual,

$$
\begin{gathered}
(n+2-2)+(n+1-2)+((n+1) / 2-2) \\
\leq\left|\left[\left\{x_{1}, x_{2}\right\}, G-\left\{y, y^{\prime}\right\}\right]_{G}\right|+\left|\left[y, G-\left(C_{1} \cup\left\{x_{1}, x_{2}\right\}\right)\right]_{G}\right| \leq 2 n .
\end{gathered}
$$

If $\delta_{2} \geq 1$, there is a $y^{\prime} \in C_{2}$ with $\left|\left[y^{\prime}, G-C_{2}\right]_{G}\right| \geq \frac{n+1}{2}$ and we get, as usual,

$$
\begin{gathered}
n+(n-1)+\left(\frac{n+1}{2}-3\right) \\
\leq\left|\left[\left\{x_{1}, x_{2}\right\}, G-\left\{y, y^{\prime}\right\}\right]_{G}\right|+\left|\left[y, G-\left(C_{1} \cup\left\{x_{1}, x_{2}, y^{\prime}\right\}\right)\right]_{G}\right| \leq n+2 \frac{n-1}{2} .
\end{gathered}
$$

Both inequalities imply that $n \leq 5$, which contradicts the relation $2=d \leq(n-3) / 2$ obtained from (14).

Finally we have the case $k=1$ with $V_{>n}\left(G_{0}\right)=\{x\}$, say. Then we also have $m=d=1$. If we had $\left|[z, x]_{G}\right| \leq(n-1) / 2$ then we would have $\left|\left[z, c_{1}\right]_{G_{0}}\right| \geq(n+1) / 2$, and hence $\left|\left[c_{1}, x\right]_{G_{0}}\right| \leq(n-1) / 2$, so $d(x) \leq n-1$. This contradiction shows that $\left|[z, x]_{G}\right| \geq(n+1) / 2$, hence $\delta \geq 1$ and, by (10), $\delta_{1} \geq 1$. Then there is a $y \in C_{1}$ with $\left|\left[y, C_{0}\right]_{G}\right| \geq(n+1) / 2$. This implies that $\left|[y, z]_{G}\right| \geq(n-1) / 2$, and hence $\delta=2$. But then we have $\delta_{1}=2$ by (10), and there is a $y^{\prime} \in C_{1}$ with $\left|\left[y^{\prime}, C_{0}\right]_{G}\right|=(n-1) / 2$. Since $n=d(z) \leq\left|[z, y]_{G}\right|+\left|[z, x]_{G}\right|$, we conclude that $\left[y^{\prime}, C_{0}\right]_{G}=\left[y^{\prime}, x\right]_{G}$, and hence $(n-1) / 2=\left|\left[y^{\prime}, x\right]_{G}\right| \leq 1$. This contradiction $n \leq 3$ completes the proof of Theorem 1.1.

Let us now turn to Theorem 1.2, whose proof is settled by Lemma 2.2 and the following lemma.

Lemma 2.3. Let $G \in \mathscr{F}_{n}$ be a simple graph with an edge of order exceeding $n$. Then

$$
|G|_{n} \geq \frac{n-1-\epsilon_{n}}{2 n+1}|G|+2+2 \epsilon_{n}
$$

Proof. There is an edge $\left[z_{1}, z_{2}\right]$ in $G$ of order at least $n+1$. Since $G$ is minimally $n$-edgeconnected, there is a cut $S$ of $G$ containing $\left[z_{1}, z_{2}\right]$. Define $C_{i}:=C\left(z_{i} ; G-S\right)$ for $i=1,2$. The graph $G_{i}$ may arise from $G$ by contracting $C_{i}$ to $z_{i}$ for $i=1,2$. Then $G_{i} \in \mathscr{F}_{n}$ and 
Theorem 1.1 provides

$$
\left|G_{i}\right|_{n} \geq \frac{n-1-\epsilon_{n}}{2 n+1}\left(\left|G_{i}\right|-\delta\left(G_{i}\right)\right)+2+\epsilon_{n}
$$

for $i=1,2$. Addition of these inequalities supplies

$$
|G|_{n}=\left|G_{1}\right|_{n}-1+\left|G_{2}\right|_{n}-1 \geq \frac{n-1-\epsilon_{n}}{2 n+1}\left(|G|+2-\delta\left(G_{1}\right)-\delta\left(G_{2}\right)\right)+2+2 \epsilon_{n} .
$$

So it only remains to show that $\delta\left(G_{1}\right)+\delta\left(G_{2}\right) \leq 2$ for $n \geq 5$. Suppose, for instance, that $\delta\left(G_{2}\right)=2$. Then there are vertices $x, y \in C_{1}$ with $\left|\left[x, z_{2}\right]_{G_{2}}\right|=(n+1) / 2$ and $\left|\left[y, z_{2}\right]_{G_{2}}\right|=(n-1) / 2$. Since $G$ is simple, this implies that $\left|N_{G}(x) \cap V\left(C_{2}\right)\right|=(n+1) / 2$ and $\left|N_{G}(y) \cap V\left(C_{2}\right)\right|=(n-1) / 2$. Then, for every $c \in C_{2},\left|\left[z_{1}, c\right]_{G_{1}}\right| \leq 2$ holds, that is, $\delta\left(G_{1}\right)=0$ for $n \geq 5$.

\section{Concluding remarks}

The lower bound for $|G|$ in Theorem 1.2 is best possible, as shown by $K_{3,5}$ for $n=3$ and $K_{n, n+1}$ for $n \geq 5$.

It remains an open question whether Theorems 1.1 and 1.2 are also best possible for $n \equiv 1 \quad(\bmod 4)$, but I doubt it, especially given my experience with the even case.

If one proceeds in a similar way for $n$ even as for $n$ odd, one comes to the conjecture that for even $n$ the slope of a best linear bound for $|G|_{n}$ is $\left(n-1 / 2-\epsilon_{n}\right) /(2 n+1)$ with $\epsilon_{n}=(3 n-2) /\left(2 n^{2}+2 n+4\right)$. That is indeed true for $n=2$, as shown in [9], but it is false for $n \geq 4$. For $n \geq 4$, a best factor must have a value lying strictly between $(n-1) /(2 n+1)$ and $\left(n-1 / 2-\epsilon_{n}\right) /(2 n+1)$. For $n=4$, I have got the exact value $8 / 23$ instead of the value $8 / 22$ above. At the moment, I have no conjecture for the exact value in the case $n$ even.

\section{References}

[1] Bollobás, B., Goldsmith, D. L. and Woodall, D. R. (1981) Indestructive deletions of edges from graphs. J. Combinatorial Theory (B) 30 263-275.

[2] Cai Mao-Cheng (1992) A remark on the number of vertices of degree $k$ in a minimally $k$-edge-connected graph. Discrete Math. 104 221-226.

[3] Cai Mao-Cheng (1993) The number of vertices of degree $k$ in a minimally $k$-edge-connected graph. J. of Combinatorial Theory (B) 58 225-239.

[4] Lick, D. R. (1972) Minimally $n$-line-connected graphs. J. Reine Angew. Math. 252 178-182.

[5] Mader, W. (1971) Minimale $n$-fach kantenzusammenhängende Graphen. Math. Ann. 191 21-28.

[6] Mader, W. (1974) Kantendisjunkte Wege in Graphen. Monatshefte für Mathematik 78 395-404.

[7] Mader, W. (1978) A reduction method for edge-connectivity in graphs. Annals of Discrete Mathematics 3 145-164.

[8] Mader, W. (1979) Zur Struktur minimal $n$-fach zusammenhängende Graphen. Abh. Math. Sem. Universität Hamburg 49 49-69.

[9] Zhu Biwen (1983) Critically 2-edge-connected graphs. Acta Math. Appl. Sinica 6 292-301. 